\title{
Effect of urea and tmao on lipid bilayers
}

Abstract We study the effect of the osmolytes, Urea and trimethylamine-N-oxide (TMAO) on POPE (1-palmitoyl-2-oleoyl-sn-glycero-3phosphoethanolamine) lipid membranes using SAXS/WAXS and DSC. Their antagonist effect is observed with TMAO stabilizing and Urea destabilizing the lipid bilayer, as seen by others in earlier researches.

Keywords lipid membranes - phase transitions-osmocytes - X-rays - DSC

\section{INTRODUCTION}

Lipid bilayers display a wide range of morphologies (di Vitta et al., 2013; Funari et al., 2009, 2011; Silva et al., 2013; Valerio et al., 2012) and are simple models for the cell membrane. They not only provide a matrix for anchoring a variety of substances, for example, membrane proteins, glycolipids and so on, that play an essential role in the cell metabolism, but also define the cell limits keeping apart the inner and outer environments. Recently, we studied the structural effects of synthetic quinones on lipid model membranes in order to investigate their contribution to morphologies, possibly involved in transfer processes. Summarizing, we can say that the insertion of these additives lowers the temperature of the structural phase transitions, and in many cases, induce the formation of cubic phases at low temperatures, ${ }^{[1,2]}$ which corresponds to an increase in the lipid matrix surface curvature.

It is accepted that urea and (TMAO) have antagonistic effects on the fluidity of lipid membranes (Barton et al., 1998; Meersman \& Walsh, 2011; Seibel et al., 2002). In red blood cells, urea slightly increases the gel-phase domains, but this effect is counteracted by TMAO. We intend to determine how these solutes affect the lipid membrane and determine their contribution to fluidity, or curvature, induced on them.

\section{OBJECTIVES}

We intended to study the influence of natural osmolytes urea and TMAO on the fluidity and stability of lipid model membranes by determining the structure of the phases formed in the lipid dispersions. In this way, we expect to determine whether a dominant interaction of these solutes takes place on the lipids, or more indirectly, by affecting the properties of the aqueous region near the headgroup surface. Small and wide angle X-ray scattering (SAXS/WAXS) thermal scans enable us to determine the number of phases in each system, their transitions and respective reversibility. SAXS/WAXS identifies these structures and provide the dimensions of their lattices. Differential scanning calorimetry (DSC) provides the phase transition temperatures and the respective energy involved in such processes.

\section{MATERIALS AND METHODS}

The samples were prepared by mixing desired volumes of stock solutions with well-defined concentrations of its components. The aqueous media at a temperature above the lipid main phase transition was directly added to the lipid. The samples were agitated slowly for two hours before they were filled into glass capillaries of $1.5 \mathrm{~mm}$ diameter and sealed. All chemicals were commercially available in high purity (POPE: Avanti lipids, TMAO and UREA : Sigma) and used without further purification.

At the SAXS beamline of ELETTRA, an in-line microcalorimeter built by the group of Michel Ollivon (CNRS, Paris, France) (M. Ollivon et al., 2006) was used to simultaneously 
measure SAXS/WAXD and high sensitivity DSC from the same sample. The calorimeter was programed to heat and cool the sample with a rate of $1^{\circ} \mathrm{C} / \mathrm{min}$ during the thermal scans between 15 and $85^{\circ} \mathrm{C}$. The acquisition time was $5 \mathrm{~s}$ each minute, and monochromatic synchrotron radiation of $0.154 \mathrm{~nm}$ wavelength was used. SAXS was monitored with a bi-dimensional Pilatus $1 \mathrm{M}$ detector positioned $c a .1 \mathrm{~m}$ away from the sample. WAXS was measured with a Pilatus $100 \mathrm{~K}$ detector at a sample to detector distance of ca. $15 \mathrm{~cm}$.

\section{RESULTS AND DISCUSSION}

Thermal scans on lipid matrices prepared with aqueous solutions of Urea or TMAO have shown morphologies similar to the ones observed in aqueous solution of the pure lipids, although with different dimensions and transition temperatures. In POPE, we observe structures with high curvature, and upon temperature cycling, the induction of cubic phases (see Figure 1), whose structure could not be uniquely identified due to the lack of resolution or a significant number of diffraction peaks originating from the samples. We note the absence of independent SAXS-based data available in the literature for the structural effect of such solutes. Some salts favour the formation of cubic phases in POPE, as described elsewhere (Tenchov \& Koynova, 2012).

A fully hydrated sample prepared in 400 mM TMAO shows the expected transitions normally seen in POPE. However, surprisingly there is a significant absence of diffraction over a wide range of temperatures on cooling from the hexagonal phase, and the diffraction peaks in the last patterns have a much lower intensity as compared to the ones at the beginning of the scan, as shown in fig 2 . The DSC traces on heating and cooling indicate phase transitions on heating at temperatures higher than for pure aqueous POPE, while during cooling this is not seen (Figure 3 ). We note that some signals were too weak and therefore not subject to our indepth interpretation. The high temperatures of the phase transition on heating indicate a stabilization of the phases.

Together with the observation that some samples have an 'oily' appearance, we conclude that TMAO hinders the self-assembly of such lipid once it interacts, most likely via hydrogen bonds, with the PE head groups. The interactions seem to become more effective at high temperatures and do not vanish easily or fast enough at our time scale during cooling. This finds support in the study on 'Hydrophobic association in mixed urea-TMAO solutions' by Ganguly et al. (2016).

Urea 200mM has a milder effect on the POPE dispersion, see fig 4. The DSC trace shows a transition at a temperature higher than for pure POPE and POPE in $400 \mathrm{mM}$ TMAO,

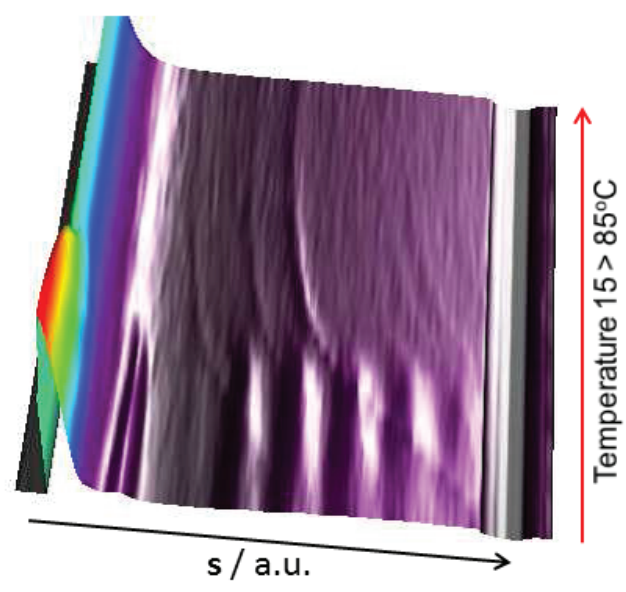

Figure1. Heating SAXS scan (15 -> 85 oC) of a POPE dispersion in solution of TMAO:Urea (2:1). The sample has been through 5 temperature cycles before this measurement. We note the occurrence of several peaks at very low angles (below the known peak of the lamellar phase). Near the beam stop (left) one sees other peaks very close to each other whose interpretation is still unclear at this point.

however involving less heat than the previous system. The thermal SAXS scan indicates a less organized stack of bilayers. The hexagonal phase, normally following the lamellar upon heating, could not be seen up to the highest temperatures of $85^{\circ} \mathrm{C}$. Moreover, no organized structure was formed during the whole temperature range during cooling. This is clear evidence that Urea has a destabilizing effect on the formed structures due to the interaction on the headgroup interface region of the bilayers assembly.

\section{CONCLUSIONS}

On combining SAXS/WAXS and DSC results obtained from dispersions of POPE in either aqueous solution of TMAO or Urea, one can see their antagonistic effect from a structural perspective, that is, their influence on the macro organization of the self-assembled mesogenic units.

Both osmolytes seem to require high temperatures (or weaker interactions between headgroups of neighbouring lipids) to be able to effectively interact with the POPE (headgroups) and exert their influence on the organization of the dispersion. TMAO favours the lamellar layer structures, while urea tends to hinder the macro organization of the system. 

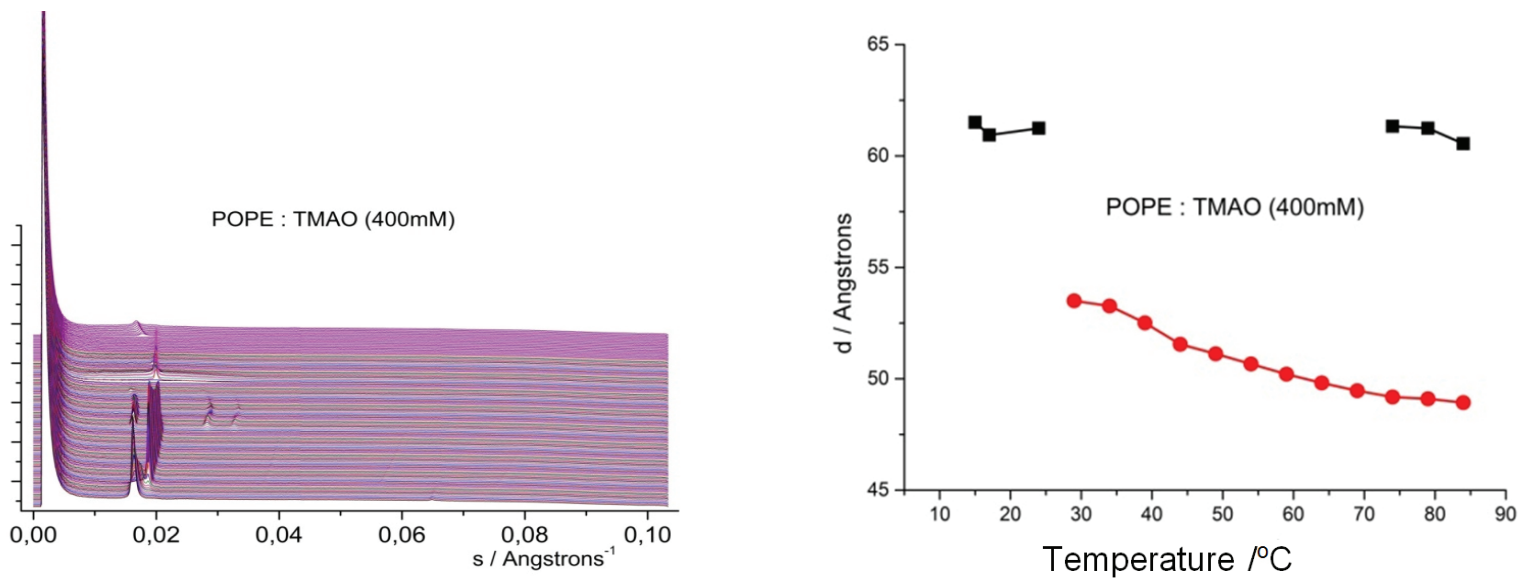

Figure 2. Sequence of diffraction patterns of POPE/TMAO. Left: Temperature scan from 15 -> 85 -> 15oC. Right: Measured spacings (thermal evolution upon heating), $d$.

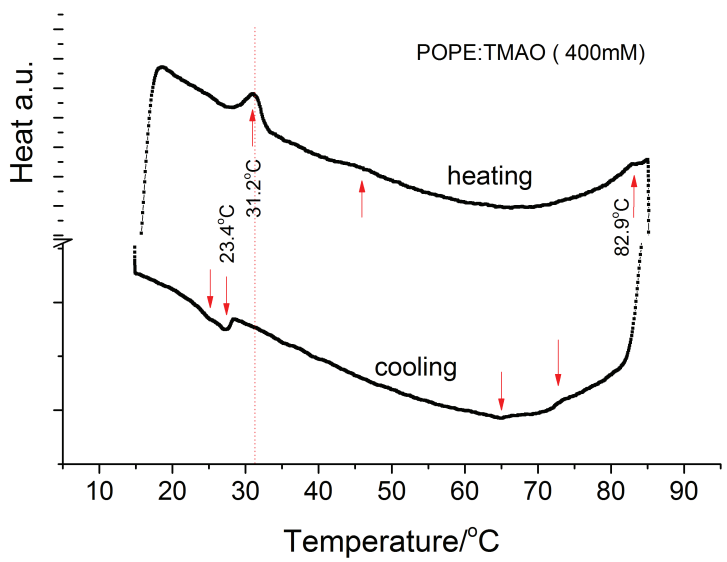

Figure 3. DSC trace of a dispersion of POPE in $400 \mathrm{mM}$ TMAO. The arrows indicate phase transition signals, many too faint to be considered for in-depth analysis.
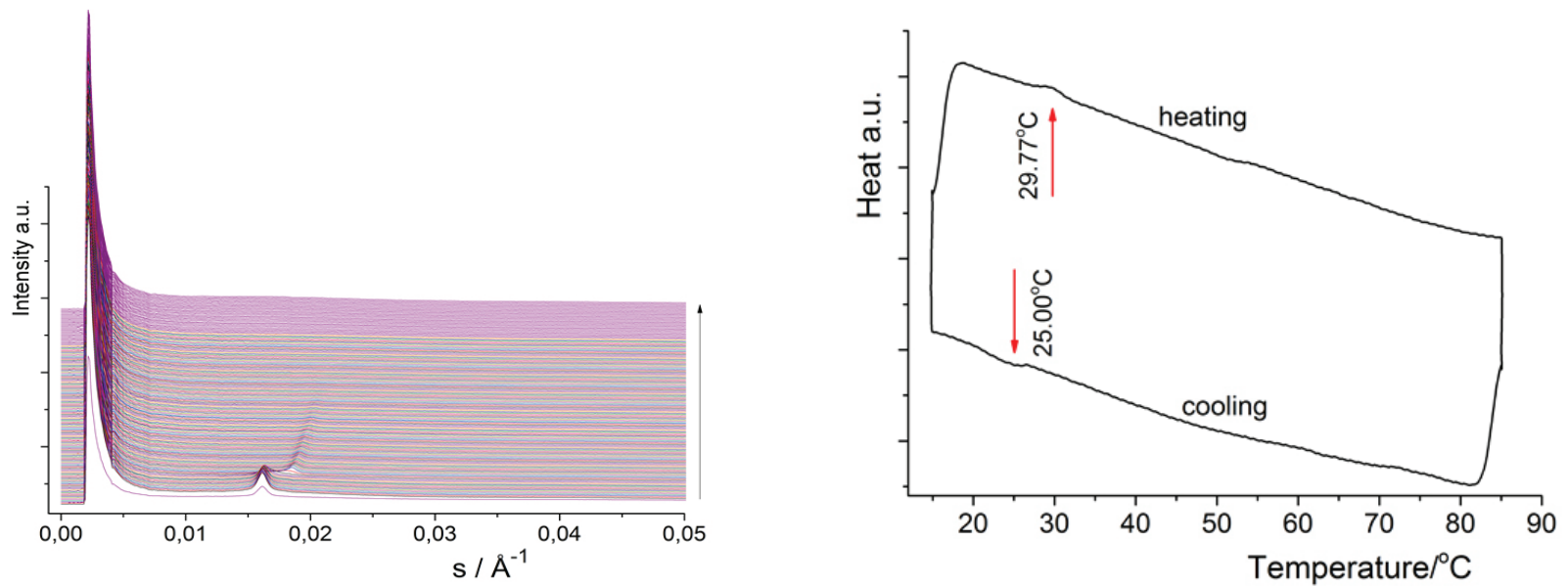

Figure 4. Thermal SAXS scan of a POPE:Urea 200mM dispersion (left) and the corresponding DSC trace (right). 


\section{Effect of urea and tmao on lipid bilayers}

\section{References}

[1] Di Vitta C, Marzorati L, Funari SS. Modification of Phospholipid Bilayers Induced by Sulfurated Naphthoquinones. J. Lipids, 2013, Article ID 592318

[2] Funari SS, Rebbin V, Marzorati L, Di Vitta C. Membrane morphology modifications induced by hydroquinones. Langmuir, 2011, 27 (13), 8257-8262.

[3] Funari SS, Rapp G, Richter F. Double-bilayer: a new phase formed by lysophospholipids and the corresponding fatty acid. Quim. Nova, 2009, 32(4), 908-912.

[4] Valerio J, Lameiro HM; Funari SS, Moreno MJ, Eurico E.Temperature Effect on the Bilayer Stacking in Multilamellar Lipid Vesicles. J. Jhys Chem. B, 2012, 116(1), 168-178.

[5] Silva T, Adao R, Nazmi K, Bolscher JGM, Funari SS, Uhrikova $D$, Bastos M. Structural diversity and mode of action on lipid membranes of three lactoferrin candidacidal peptides. Biochim Biophys Acta, 2013, 1828(11), 2796-2796.

[6] Barton KN, Buhr MM, Ballantyne J.S. Effects of urea and trimethylamine $\mathrm{N}$-oxide on fluidity of liposomes and membranes of an elasmobranch; Am. J. Physiol.-Regulat. Integ. Comp. Physiol. 1998, 276, R397-R406.
[7] Seibel BA, Walsh PJ. Trimethylamine oxide accumulation in marine animals: relationship to acylglycerol storage. J. Exp. Biol. 2002, 198, 373-378.

[8] Meersman F, Bowron D, Soper AK, Koch MHJ. An X-ray and neutron scattering study of the equilibrium between trimethylamine $\mathrm{N}$-oxide and urea in aqueous solution. Phys. Chem. Chem. Phys.2011, 13(30), 13765-13771.

[9] Tenchov B \& Koynova R. Cubic phases in membrane lipids. Eur Biophys J (2012),41:841.

[10] Ganguly P, van der Vergt NFA Shea JE. Hydrophobic association in mixed urea-TMAO solutions, J. Phys. Chem. Let. 2016, 7(15), 3052-3069.

[11] Ollivon M, Keller G, Bourgaux C, Kalnin D, Villeneuve P, Lesieur P., J. Therm Anal Calorim; DSC and high resolution $\mathrm{X}$-ray diffraction coupling ; Therm. Anal. Calorim. 2006, 85(1), 219. 